\title{
Cross-Generational Effects and Non-random Developmental Response to Temperature Variation in Paramecium
}

\author{
Rebecca Hagen, Valerio Vitali and Francesco Catania* \\ Department of Biology, Institute for Evolution and Biodiversity, University of Münster, Münster, Germany
}

\section{OPEN ACCESS}

Edited by:

Alessandro Minelli,

University of Padua, Italy

Reviewed by:

Douglas Lee Chalker, Washington University in St. Louis,

United States

Patricia Johnston Moore, University of Georgia, United States

*Correspondence:

Francesco Catania francesco.catania@uni-muenster.de

Specialty section:

This article was submitted to Evolutionary Developmental Biology, a section of the journal Frontiers in Cell and Developmental

Biology

Received: 16 July 2020 Accepted: 30 September 2020 Published: 20 October 2020

Citation:

Hagen $R$, Vitali $V$ and Catania $F$ (2020) Cross-Generational Effects and Non-random Developmental Response to Temperature Variation

in Paramecium.

Front. Cell Dev. Biol. 8:584219. doi: 10.3389/fcell.2020.584219
Unicellular organisms such as ciliates are largely neglected in research on adaptive developmental plasticity, although their nuclear dualism offers ideal circumstances to study development outside an embryonic context. Here, we gain first insights into the ability of the ciliate Paramecium to develop potentially adaptive phenotypic changes in response to early-life adversity. We show that, upon exposure to unconventional culture temperatures, germ line-to-soma differentiation gives rise to coordinated molecular changes that may help attune the number of functional gene copies to the new external conditions. The non-random somatic heterogeneity that developmental plasticity generates is largely epigenetically controlled, shaped by the parental experience, and may prompt a stress response. These findings establish Paramecium as a new model system to study the molecular basis and evolutionary significance of developmental plasticity. In echoing previous indications in mammals, they call for an incorporation of intergenerational effects in adaptation studies.

Keywords: programmed DNA elimination, adaptive developmental plasticity, temperature, genome evolution, Paramecium, intergenerational effects, epigenetics

\section{INTRODUCTION}

Environmental cues affecting organismal development may produce long-term patterns of gene expression and induce novel phenotypes as life advances (Beldade et al., 2011; Cole et al., 2012; Tung et al., 2015). These phenotypes may or may not be associated with disease, and may be passed on to the next sexual generation(s) via epigenetic mechanisms. Thus, developmental plasticity (henceforth referred to as plasticity) may contribute both to diseases and the onset of evolutionary adaptations. Despite the seemingly straightforward ways of testing this possibility, whether and how plasticity truly affects health and/or evolution are still matters of contention.

Existing models on the role of plasticity in health and evolution make different, even conflicting predictions. Regarding health, plasticity may either enhance survival in early life at the expense of adult health (developmental constraints model), or prepare an individual to respond optimally to an anticipated environment later in life (predictive adaptive response model) (Nettle and Bateson, 2015; Lea et al., 2017). Both developmental constraints and predictive models assume that plasticity evolved through natural selection (Watve, 2017). However, only the predictive model seems to maintain that individuals that are exposed to low-quality environments in early life will respond adaptively later in life by maximizing reproduction within their expected shorter lifespan 
(Wells, 2012). As for the significance of plasticity in evolution, evidence from natural populations suggests that adaptive and non-adaptive plasticity can both hinder and facilitate adaptation to new environments (e.g., Ghalambor et al., 2015) vs. (Scoville and Pfrender, 2010). These seemingly contradictory observations build on substantial theoretical research and a relatively small number of empirical studies, an insufficiency that is, in part, attributable to historical skepticism toward the significance of plasticity for evolution (Sommer, 2020). Experimental evolution studies in natural settings may be viewed as the best way to obtain valuable insights into how evolution proceeds in nature. However, these studies have also some disadvantages. For example, they may occur in complex ecosystems and unfold over long time periods, circumstances that may make it difficult to control for all relevant ecological factors contributing to selection pressures. New tractable model systems where the evolutionary significance of plasticity can be easily and rapidly tested under controlled laboratory conditions would be highly valuable to evolutionary biologists. These systems may help in identifying conserved molecular mechanisms that influence developmental reprogramming in response to the environment, and provide insights into how to integrate the roles that plasticity plays in health and evolution.

To date, virtually all of the studies on plasticity have focused on multicellular organisms (Gluckman et al., 2009; Beldade et al., 2011; Bateson et al., 2014; Lea et al., 2017; Lu et al., 2019). This is because development is commonly viewed as an embryonic or cellular process. However, development may also take place at the sub-cellular level. Ciliates such as the free-living Paramecium tetraurelia (henceforth referred to as Paramecium) offer a spectacular example of nuclear development. Paramecium contains a diploid germ line (micro)nucleus and a highly polyploid somatic (macro)nucleus in a single cell. Unlike the germ line micronucleus, the somatic macronucleus is transcriptionally active during the cell's vegetative life and determines its phenotype. At each sexual cycle, the macronucleus degrades slowly and a new (filial) somatic macronucleus differentiates from a mitotic copy of the zygotic nucleus (Coyne et al., 1996, 2012; Betermier and Duharcourt, 2014) (Figure 1).

It has become increasingly clear that Paramecium biology is close to that of many other eukaryotes. For example, sex, stress response, growth, and lifespan are mechanistically linked in Paramecium as they are in multicellular eukaryotes (Arking, 2018). For example, low-quality food accelerates sexual maturation in Paramecium (Thind et al., 2020), and the timing of sexual maturity in this microbial eukaryote positively correlates with lifespan (Smith-Sonneborn, 1981). At the same time, Paramecium possesses biological properties that are conveniently different from those of multicellular and long-lived organisms that are commonly used to investigate adaptive plasticity. Indeed, as Paramecium can be grown in the lab for successive sexual generations without essentially acquiring new germ line mutations (Sung et al., 2012; Long et al., 2018), the shortand long-term effects of plasticity on the fitness of isogenic lines can be readily distinguished and assessed. All of this makes Paramecium an ideal system by which to advance general understanding of the complex interplay between epigenetics and genetics and to generate hypotheses on the effect of development on adaptation that can be tested in less amenable systems.

Furthermore, empirical findings in Paramecium might help further advance current understanding of plasticity in multicellular systems. In one example, studies on Paramecium could help assess whether adaptive plasticity is only triggered in response to environments that the organism has recurrently experienced over evolutionary time. Studies on Paramecium could also expand current views and generate fresh hypotheses in metazoans. For example, the timing of sexual maturity in Paramecium is reduced not only by the low quality of the environmental conditions (Thind et al., 2020) but also as the age of the parental cells increases (Siegel, 1961; Takagi et al., 1987). Thus, in addition to early life environments, parental experience may affect the phenotypic state of an individual. Incorporating parental effects in current explanations for plasticity in metazoans is essential to gain a more accurate perspective of the role of plasticity in evolution (Wells, 2017).

Nuclear development in Paramecium generates molecular variation (Duret et al., 2008; Catania et al., 2013), more so when Paramecium faces new environmental conditions (Vitali et al., 2019). This somatic genome plasticity is, at least in part, the byproduct of a perturbed developmental process known as programmed DNA elimination (PDE). In Paramecium, PDE operates at the genome-wide level and eliminates $\sim 45,000$ intervening DNA segments-Internal Eliminated Sequences (IESs) - during the development of a new somatic macronucleus (Arnaiz et al., 2012) (Figure 1). Although this elimination is largely faithful, PDE may fail to remove some IESs from all the copies of the newly developing somatic genome. Thus, 100 s of Paramecium IESs may accumulate in the new somatic macronucleus at each sexual cycle, with a variable fraction of somatic DNA copies affected by the IES incorporation.

Incomplete IES excisions are commonly regarded as errors with limited biological significance. However, previous studies have found signatures of purifying selection antagonizing IES retention (Arnaiz et al., 2012; Ferro et al., 2015; Vitali et al., 2019). This suggests that incomplete IES excision in Paramecium may have non-trivial phenotypic consequences. Furthermore, although the somatic nucleus is replaced at each sexual cycle, somatic IESs may nonetheless be transmitted to the sexual offspring via RNA-mediated epigenetic mechanisms (Duharcourt et al., 1995, 1998, 2009). These mechanisms are similar to those that contribute to the trans-generational transmission of parental phenotypic responses in animals, fungi, and plants (Duempelmann et al., 2020). Thus, epigenetic mechanisms in Paramecium may enable the inheritance of somatic IESs and their potential phenotypic effects-these changes might later become genetically encoded. Further, conserved RNA-mediated molecular dynamics may underlie the evolutionary significance of plasticity across developmental systems, bridging the gap between ciliates and metazoans.

Here, we consider the process of germ line to soma differentiation in the single-celled Paramecium and characterize the relationship between this developmental process's plasticity and somatic gene expression (a proxy for phenotypic variation). We uncover coordinated and predictable somatic changes, 


\section{NUCLEAR DEVELOPMENT}

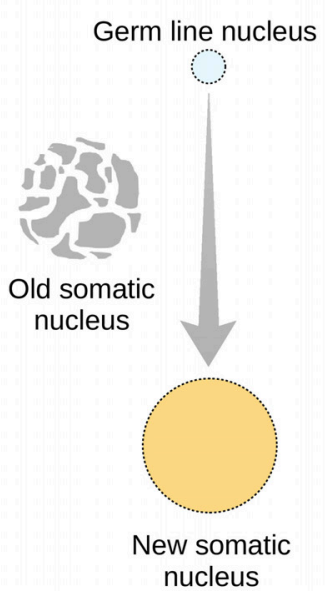

PROGRAMMED DNA ELIMINATION

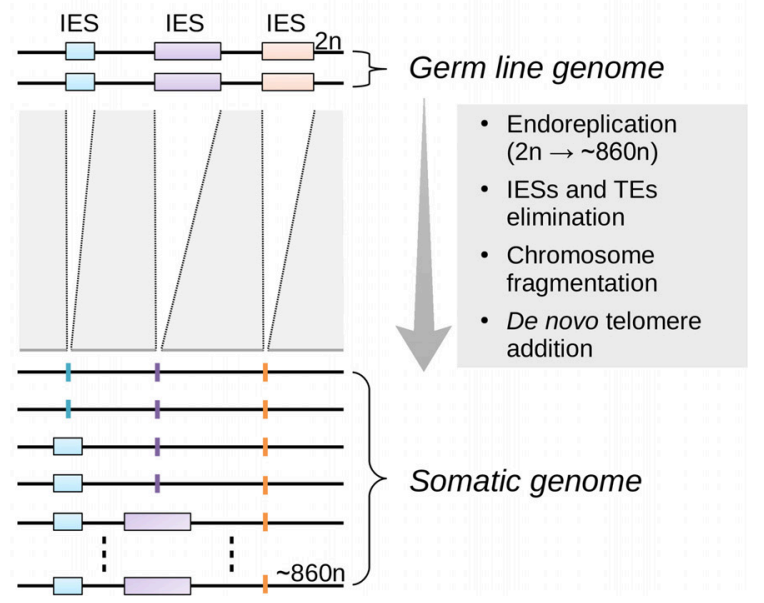

FIGURE 1 | Nuclear replacement and somatic genome development in Paramecium. Each time the single-celled Paramecium reproduces sexually, the old (parental) somatic nucleus is destroyed and replaced with a new one (left). The new macronucleus develops from a copy of the zygotic micronucleus housing the germ line genome. The process of genome remodeling that accompanies nuclear development in Paramecium involves endoreplication, chromosome fragmentation and de novo telomerization, and the elimination of mostly germ line-restricted elements such as transposable elements (TEs) and Internal Eliminated Sequences (IESs) (right). Only IES elimination is shown. This developmentally-regulated genome processing is known as Programmed DNA Elimination (PDE). PDE is crucial to reconstitute a functional somatic genome from an interrupted germ line template.

which allow causally linking the environments that Paramecium experiences during its vegetative life/somatic development to the number of productive somatic gene copies in its sexual offspring. In extending our previous work (Vitali et al., 2019), these findings empirically connect ecology and development in a microbial eukaryote while offering insights into the molecular processes that underlay plasticity.

\section{MATERIALS AND METHODS}

\section{Paramecium Stocks, Culture Conditions, Published and Newly Generated Genome Datasets}

We re-examined the IES excision profiles of fully homozygous lines of $P$. tetraurelia stocks 51 and $\mathrm{d} 12$, which were generated in previously published experiments (Arnaiz et al., 2012; Lhuillier-Akakpo et al., 2014; Swart et al., 2017; Vitali et al., 2019). P. tetraurelia stocks 51 cells were cultured at $27^{\circ} \mathrm{C}$ during both vegetative growth and nuclear differentiation, when somatic genome development/Programmed DNA Elimination takes place (Arnaiz et al., 2012; Lhuillier-Akakpo et al., 2014; Swart et al., 2017). A fully clonal population of $P$. tetraurelia stocks $\mathrm{d} 12$ was cultured at $25^{\circ} \mathrm{C}$ (F0) and subjected to 18 , 25 , or $32^{\circ} \mathrm{C}$ (F1) during somatic genome development and the successive vegetative phase, when cells divide asexually. These lines were generated in Vitali et al. (2019) to investigate the impact of different environmental temperatures on somatic genome development. One additional F1 line (referred to as $25^{\circ} \mathrm{C}_{F 1}^{*}$ in the main text) was also generated in Vitali et al. (2019), but analyzed here for the first time. Line $25^{\circ} \mathrm{C}^{*} \mathrm{~F} 1$ originates from the same parental population that fathered lines $18^{\circ} \mathrm{C}_{F 1}, 25^{\circ} \mathrm{C}_{F 1}$, and $32^{\circ} \mathrm{C}_{F 1}$ and like $25^{\circ} \mathrm{C}_{F 1}$ was subjected to $25^{\circ} \mathrm{C}$ during nuclear differentiation. However, the parental cells of $25^{\circ} \mathrm{C}^{*}{ }_{\mathrm{F}}$ were additionally subjected to a $40^{\circ} \mathrm{C}$ heat shock for $30 \mathrm{~s}$ daily during their vegetative life. Line $25^{\circ} \mathrm{C}_{F 1}^{*}$ and $25^{\circ} \mathrm{C}_{F 1}$ were used to test the impact of ecological factors experienced by the parental population on a future instance of somatic genome development. The set of treatments applied to the fully clonal parental population of $P$. tetraurelia stock $\mathrm{d} 12$ are summarized in Figure 2. For each of the lines surveyed, isogenic cells were expanded to mass culture for somatic DNA extraction (as previously described Arnaiz et al., 2012; Lhuillier-Akakpo et al., 2014; Swart et al., 2017; Vitali et al., 2019). Culture conditions, macronuclear DNA isolation, whole-genome sequencing and data preprocessing for the $25^{\circ} \mathrm{C}_{F 1}^{*}$ sample are as previously described (Vitali et al., 2019).

\section{IES Datasets Analyses}

The software ParTIES (Denby Wilkes et al., 2016) was used to estimate IES Retention Scores (IRSs), i.e., the per-locus ratio between IES-containing reads and the total number of mapping reads. IES loci with significantly different retention levels between F0 and F1 lines were designated as previously described (Vitali et al., 2019). Namely, the upper and lower bound of the 75\% confidence interval constructed on the F0 retention score was taken as a reference retention score for binomial testing of upward or downward transitions, respectively. IES loci supported by $<20$ sequence reads were excluded and only IESs with a size larger than 25 nucleotides are considered for this study. The sets of IESs whose excision is epigenetically controlled 


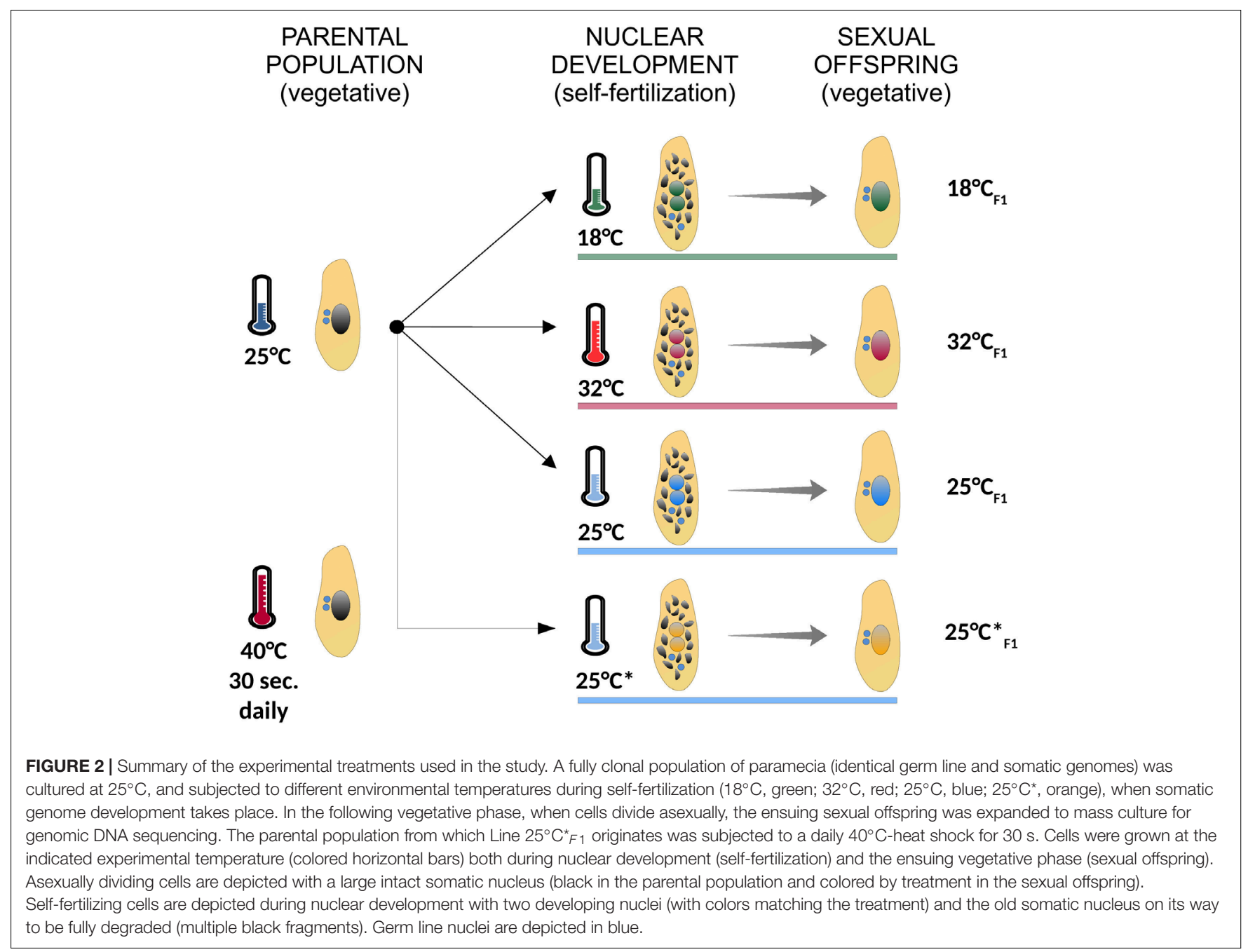

in $P$. tetraurelia stock 51 were obtained from ParameciumDB (Arnaiz and Sperling, 2011).

\section{Gene Expression Data and GO Term Enrichment}

Transcriptomes were previously obtained for $P$. tetraurelia stocks 51 (Arnaiz et al., 2017) and d12 (Vitali et al., 2019), using cells at the vegetative stage cultured at standard conditions $\left(25-27^{\circ} \mathrm{C}\right)$. High and weak gene expression level categories are based on whether the expression values fall into the first or the last quartile of an underlying log2transformed distribution. The functional enrichment analyses of somatic-IES containing genes were performed using the functional annotation tool DAVID (Huang et al., 2009a,b). As reference set for the genes that harbor DCL2/3-sensitive, PTC-inducing, and exon-mapping small IESs and are highly expressed at standard conditions, we used genes that harbor small exon-mapping IESs. As reference set for the genes that are weakly expressed at standard conditions and that harbor DCL5-sensitive, PTC-inducing, large and exon-mapping IESs, we used genes that harbor large exon-mapping IESs. In all cases, Fisher's exact test estimates the over-representation of GO-terms.

\section{Data Analysis}

All the analyses were conducted using R Core Team (2018).

\section{Data Access}

High-throughput sequencing data generated for the $25^{\circ} \mathrm{C}^{*} \mathrm{~F} 1$ sample have been submitted to the European Nucleotide Archive ${ }^{1}$ under the accession number ERR4179861.

\section{RESULTS AND DISCUSSION}

\section{A Relationship Between PDE Efficiency and Somatic Gene Expression Level}

We asked whether vegetative gene expression levels and PDEmediated developmental variation are linked in Paramecium. PDE removes Internal Eliminated Sequences (IESs) from the

\footnotetext{
${ }^{1}$ https://www.ebi.ac.uk/ena
} 
developing polyploid somatic genome and this removal can be incomplete. The per-locus magnitude of somatic IES retention is approximated by the relative fraction of IES-containing sequence reads and is termed IES Retention Score (IRS $=0$, no retention; IRS $=1$, full retention).

Using previously published transcriptomic data (Arnaiz et al., 2017) and two independent sets of incompletely excised IESs from the same Paramecium stock cultivated in standard conditions (Arnaiz et al., 2012; Lhuillier-Akakpo et al., 2014), we find that gene expression levels correlate negatively with IRS estimates (Kendall's tau $\leq-0.127, P<0.0001$ ). The high statistical significance of this observed negative relationship between IRS and gene expression levels holds when single-copy genes and genes with duplicates are examined separately. As differently sized IESs may be subjected to different mechanisms of excision (Arnaiz et al., 2012; Ferro et al., 2015), we checked whether the statistical significance of this correlation holds for small and large IESs alike. Additionally, we inspected the distribution of non-trivial IES retentions (IRS $>0.1$ ) in genes that are weakly and highly expressed (WEG and HEG, respectively). We found that WEG harbor a statistically significant excess of somatic IESs relative to HEG (Table 1). This excess holds firmly for large IESs, but it is less pronounced for small IESs (Table 1). Furthermore, small IESs undergo $>3$ times more incomplete excision than large IESs in HEG (0.25 vs. $0.08 \%$ ), whereas they display comparable levels of incomplete excision to large IESs in WEG (0.84 vs. $0.97 \%$, respectively). Thus, the level of developmental variation in Paramecium varies with the level of somatic gene expression, and the strength of this relationship is IES-size dependent.

\section{Natural Selection Affects IES Distribution and Splicing Fidelity in the Paramecium Genome}

Two observations can help explain why WEG retain an excess of large IESs (Table 1). First, WEG in Paramecium experience lower levels of selective pressure compared to HEG (Gout et al., 2010). Second, large WEG-mapping IESs display weaker cisacting DNA-level splicing signals compared to same-size HEGmapping large IESs ( 0.60 vs. 0.64 ; Wilcoxon rank sum test, $P=0.023)$. Thus, the relatively lower selective pressure on WEG may contribute to the deterioration of large IESs' cis-acting recognition/excision signals, thereby increasing these IESs' risk of incomplete excision. As small IESs may rely less on cis-acting sequence signals compared to large IESs (Ferro et al., 2015), this explanation also accounts for the marginal over-representation of small somatic IESs in WEG (Table 1).

Rationalizing the threefold surplus of incompletely excised small IESs in HEG is less straightforward. If incomplete IES excision reflects errors and may be detrimental as commonly postulated, then natural selection is expected to purge small IESs from HEG. Consistent with this, there is a deficit of small IESs in Paramecium HEG (Ferro et al., 2015 and Table 1). But then, it is unclear why a subset of small IESs that undergo potentially hazardous incomplete elimination is found in HEG.

One possibility is that modern HEG-mapping small IESs undergo only trivial and thus tolerable levels of retention. The data at our disposal do not align well with this scenario. As illustrated in Table 1, the incomplete excision of HEG-mapping small IESs may entail $>10 \%$ of the somatic DNA copies (IRS > 0.1). Additionally, we detect a systematically higher number of small IESs with IRS $>0.1$ in HEG relative to large IESs in each of the two experiments considered above and in two other independent experiments that we revisited (Swart et al., 2017) (in total: 15 vs. 6 , respectively).

Another possible explanation for the evolutionary persistence of small IESs in HEG is that their retention has no impact on protein sequence/function. However, upon examining four independent experiments (Arnaiz et al., 2012; Lhuillier-Akakpo et al., 2014; Swart et al., 2017), we detect a significant enrichment (rather than a deficit) of premature termination codons (PTCs) induced upon retention of small IESs compared to what would be expected by chance ( 1.1 vs. $0.5 \%, P=0.009)$. We find no statistically significant enrichment in WEG instead (3.0 vs. 2.6\%, $P=0.281$ ) (note that in this latter analysis we used IESs with IRS $>0.05$, rather than IRS $>0.1$, to increase the power of the statistical test).

In sum, our observations raise the possibility that natural selection might actually favor the incomplete excision of a subset of small and PTC-inducing IESs in a variable fraction of HEG. The question then arises: is the alternative splicing of these IESs in Paramecium's HEG functional?

\section{The Count of Incompletely Excised Small Exon-Mapping IESs Increases Upon Environmental Changes}

Paramecium genes that retain PTC-inducing introns generate transcripts that normally become targets of the Nonsense Mediated Decay (NMD) pathway for degradation (Jaillon et al., 2008). Thus, the partial retention of small PTC-inducing IESs

TABLE 1 | Incomplete IES excision (IRS > 0.1) affects weakly expressed genes (WEGs) more frequently than highly expressed genes (HEGs).

\begin{tabular}{|c|c|c|c|c|c|}
\hline IESs & IESs in HEG (IRS $\geq 0$ ) & IESs in WEG (IRS $\geq 0$ ) & IESs in HEG (IRS > 0.1) & IESs in WEG (IRS > 0.1) & Two-proportion Z-test, $P^{\star}$ \\
\hline All & 8108 & 9504 & 12 & 87 & $2.3 e-11$ \\
\hline Large & 5142 & 4725 & 4 & 46 & 9.5 e-10 \\
\hline Small & 2765 & 4544 & 7 & 38 & 0.0033 \\
\hline
\end{tabular}

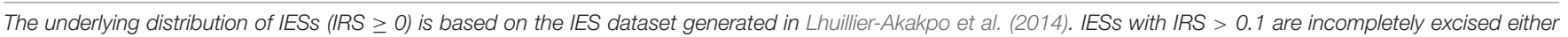

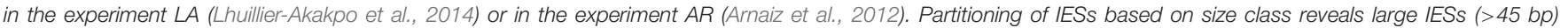

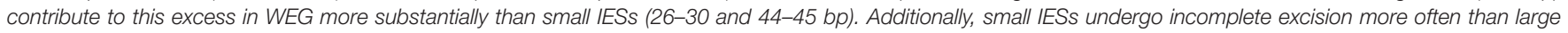
IESs in HEGs. IESs were partitioned in size groups following (Ferro et al., 2015). *No correction for multiple testing. 
in Paramecium's HEG may effectively reduce the number of productive (i.e., functional) gene copies in the somatic nucleus. This may impact the levels of somatic transcription, thereby helping rationalize the detected relationship between PDEmediated developmental variation and somatic gene expression levels. An IES-mediated regulatory function might also help Paramecium cope with environmental changes. More specifically, environmental changes during somatic development could affect the excision of small PTC-inducing IESs tuning the number of productive somatic gene copies to the new surroundings. This hypothetical scenario predicts that small HEG-mapping IESs that introduce PTCs upon incomplete excision will enter into play when Paramecium is exposed to new environments.

To test this hypothesis, we revisited a dataset generated recently (Vitali et al., 2019). We examined changes in incomplete IES excision across two consecutive sexual generations (F0 and F1) with or without changes in environmental temperature $\left(25^{\circ} \mathrm{C}_{F 0} \rightarrow 25^{\circ} \mathrm{C}_{F 1} ; 25^{\circ} \mathrm{C}_{F 0} \rightarrow 18^{\circ} \mathrm{C}_{F 1} ; 25^{\circ} \mathrm{C}_{F 0} \rightarrow 32^{\circ} \mathrm{C}_{F 1}\right)$. We find that $\mathrm{F} 0$ and $\mathrm{F} 1$ cells that are both cultivated at $25^{\circ} \mathrm{C}$ display comparable numbers of small and large somatic IESs (F1/F0 ratio: 1.3 and 0.8 for small IESs and large IESs, respectively). However, significant differences emerge when we consider F0 and F1 cells that are exposed to different temperatures. The count of somatic IESs in the $18^{\circ} \mathrm{C}_{F 1}$ and $32^{\circ} \mathrm{C}_{F 1}$ lines not only rises considerably, as previously described (Vitali et al., 2019), but is also unevenly distributed with regard to IES size and gene expression levels. Relative to the cells cultivated at $25^{\circ} \mathrm{C}, 18^{\circ} \mathrm{C}_{F 1}$, and $32^{\circ} \mathrm{C}_{F 1}$ cells contain more somatic small IESs (4 and 5 times, respectively) than large IESs ( 2 and 2.5 times, respectively) (Figure 3A). These IES size-dependent differences disappear for IESs that map to intergenic regions (Figure 3B). Furthermore, small somatic IESs in $32^{\circ} \mathrm{C}_{F 1}$ cells preferentially reside in genes that are highly expressed in the parental cells (two-proportion Z-test, $P=0.006$ ) -we detected a similar preference for $25^{\circ} \mathrm{C}_{F 1}$ (two-proportion $\mathrm{Z}$-test, $P=0.05$ ). More than $70 \%$ of these IESs
$(11 / 15)$ introduce in-frame stops based on P. tetraurelia stock 51's gene annotation.

These observations support a tentative model where IESmediated gene copy disruption mediates the down-regulation of a subset of Paramecium genes.

\section{The Magnitude of Incomplete Excision for Large Exon-Mapping IESs Decreases Upon Environmental Changes}

In the course of our analyses, we also detected a positive correlation between IES retention levels and the size of exonmapping IESs with IRS $>0.1$ at $25^{\circ} \mathrm{C}$ (Figure $4 \mathrm{~A}, 25^{\circ} \mathrm{C}_{F 0}$ : Kendall's tau $=0.188, P=0.009 ; 25^{\circ} \mathrm{C}_{F 1}$ : Kendall's tau $=0.169$, $P=0.019)$. Additionally, the average IRS of large exonmapping IESs is significantly reduced in $18^{\circ} \mathrm{C}_{F 1}$ and $32^{\circ} \mathrm{C}_{F 1}$ cells compared to the parental cells cultivated at $25^{\circ} \mathrm{C}$ (Wilcoxon rank sum test, $P<0.0001)$. Finally, F1 cells cultured at 18 and $32^{\circ} \mathrm{C}$ tend to accumulate better-excised, large and exonmapping IESs in genes that are weakly expressed in the parental environment $\left(18^{\circ} \mathrm{C}_{F 1}: 5\right.$ large vs. 2 small IESs with reduced IRS; 11 large vs. 21 small IESs with increased IRS; Fisher's exact test, $P_{\text {one-tail }}=0.085 ; 32^{\circ} \mathrm{C}_{F 1}: 7$ large vs. 2 small IESs with reduced IRS; 25 large vs. 35 small IESs with increased IRS; Fisher's exact test, $\left.P_{\text {one-tail }}=0.047\right)$.

These observations raise the possibility that the hypothetical IES-mediated regulation of productive somatic gene copy number in Paramecium may proceed not only through gene copy disruption (via small IES retention in HEG), but also through gene copy re-activation (via large IES excision from WEG). This large IES-mediated regulation may extend to intergenic regions where significant changes in IRS are recorded both for large and small intergenic IESs at $32^{\circ} \mathrm{C}$ (Wilcoxon rank sum test, $P<0.005$ ) and for small IESs at $18^{\circ} \mathrm{C}$ (Wilcoxon rank sum test, $P=0.011$ ) (Figure 4B).

\section{A}

\section{Exon-mapping IESs with IRS $>0.1$}

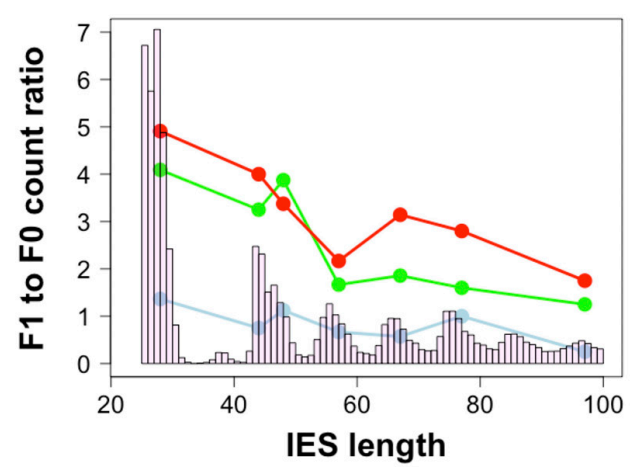

B

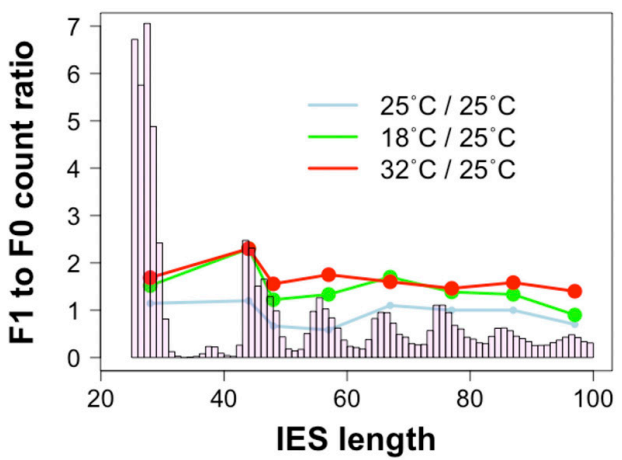

FIGURE 3 | Ratio of incomplete IES excision (IRS > 0.1) counts in parental and filial generations. The two consecutive generations are exposed to either a stable environment in which both $\mathrm{FO}$ and $\mathrm{F} 1$ were cultivated at $25^{\circ} \mathrm{C}$ (light blue) or a changing environment in which the F0 was cultivated at $25^{\circ} \mathrm{C}$, but the $\mathrm{F} 1$ was cultivated either at $18^{\circ} \mathrm{C}$ (green) or $32^{\circ} \mathrm{C}$ (red). Grouping IESs according to genomic location (coding exons, intergenic) reveals that small exon-mapping IESs are more often incompletely excised compared to larger exon-mapping IESs (A). No size-dependent pattern is detected for intergenic IESs (B). Ratios were calculated after summing incompletely excised IESs falling in the most frequent size classes (bp): 26-30, 44-45, 46-50, 54-60, 64-70, 74-80, 84-90, and 94-100. Only size classes with $\geq 3$ incompletely excised IESs per condition were examined. 
A

\section{Exon-mapping IESs with IRS $>0.1$}

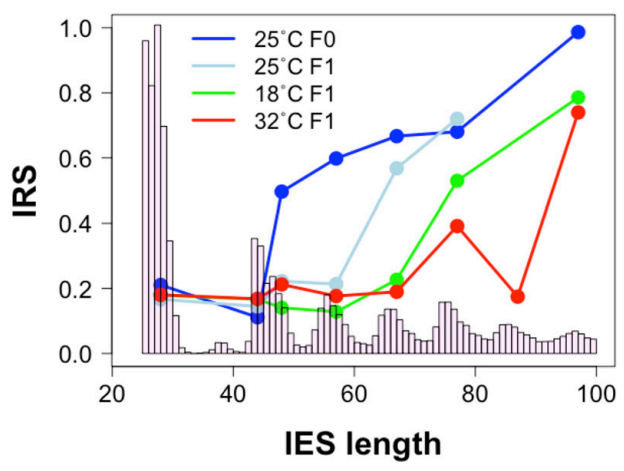

B

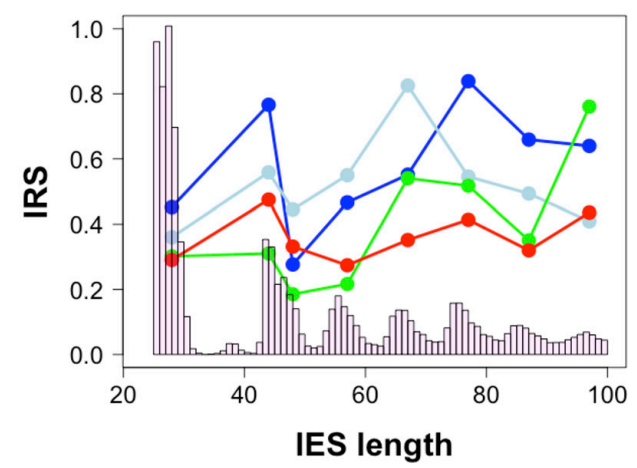

FIGURE 4 | Relationship between IES size and IES Retention Score (IRS). (A) The median IRS of small IESs, but not of large IESs, is comparable between F0 and F1 cells cultivated at $25^{\circ} \mathrm{C}$ (dark and light blue lines) and at the non-standard culture temperatures of $18^{\circ} \mathrm{C} \mathrm{(green} \mathrm{line)} \mathrm{and} 32^{\circ} \mathrm{C}$ (red line). (B) In IESs that map to intergenic regions no clear IES size-related trend is discernible. Median IRS values were calculated using incompletely excised IESs that fall in the most frequent size classes (bp): 26-30, 44-45, 46-50, 54-60, 64-70, 74-80, 84-90, and 94-100. Only size classes with $\geq 3$ incompletely excised IESs per condition were examined.

\section{IES-Mediated Changes in Functional Gene Copies May Be Epigenetically Controlled}

Because cis-acting IES recognition/excision signal sequences are highly likely to remain invariable across two consecutive sexual generations, trans-acting factors must shape the aforementioned differences in small and large IES recognition/excision efficiency. We therefore examined how extensively the putative regulated/regulatory IESs fall into a class of IESs that are under epigenetic control in the $P$. tetraurelia stock 51 (henceforth referred to as epi-IESs). Two classes of small RNAs are involved in the excision of epi-IESs in Paramecium. Two Dicer-like proteins (DCL2 and DCL3) produce scnRNAs that transit through the parental somatic nucleus from the germ line nucleus before targeting IESs for excision in the developing somatic nucleus (Lepere et al., 2009; Bouhouche et al., 2011). The Dicer-like protein DCL5 produces iesRNAs directly in the developing somatic nucleus from excised amplified IESs (Sandoval et al., 2014).

We found that small PTC-inducing IESs that map to HEG exons preferentially fall into a class of DCL2/3-sensitive IESs (Figure 5A). Instead, large exon-mapping IESs with reduced incomplete excision (i.e., reduced IRS) in F1 cells are enriched with DCL5-sensitive IESs $\left(32^{\circ} \mathrm{C}_{F 1}\right.$ : Fisher's Exact Test, $P=0.008$; Figure 5B). The limited dataset does not allow us to test whether this enrichment is specific to WEG in the parental generation.

We also leveraged the study of epi-IESs (DCL2/3-sensitive IESs, in particular) to rule out an additional interpretation of our observations. Namely, selection may have promoted the gain of PTCs in Paramecium's weakly excised and HEG-mapping IESs to solely deal with the detrimental effects of IES retention, not because IES retention changes the level of gene expression. If selection promoted PTC retention to deal with the detrimental effects of IES incomplete excision, then epi-IESs should be as likely to introduce PTCs as non-epi-IESs are. However, under a model of environmentally induced and IES-mediated modulation of gene expression, epi-IESs should be more likely than non-epiIESs to contain PTCs. We found that the incomplete excision of non-epi-IESs is 2.4-fold more likely to introduce PTCs in the corresponding transcripts than to leave the ORF intact (PTC-inducing $=348$, PTC-free $=147)$. On the other hand, the incomplete excision of epi-IESs is 14 -fold more likely to introduce PTCs $($ PTC-inducing $=14$, PTC-free $=1$ ). This relative enrichment of PTC-inducing epi-IESs holds for $18^{\circ} \mathrm{C}_{F 1}$ cells ( 9 vs. 2.3 fold).

As the bias in favor of PTC-inducing epi-IESs could result from these IESs' more elevated (and therefore potentially more detrimental) level of retention compared to non-epiIESs (median IRS: 0.023 vs. 0.013 for epi- and non-epi-IESs, respectively), we reanalyzed our data after accounting for the IRS discrepancy between the two sets of IESs, i.e., upon considering non-epi-IESs with IRS $>0.023$. We found that the incomplete excision of non-epi-IESs is still not more than $\sim 2$ fold more likely to introduce PTCs (PTC-inducing $=80$, PTC-free $=41$ ). Thus, epi-IESs are substantially more likely than non-epi-IESs to induce PTCs upon their retention.

These findings and the foregoing observations align with a model where PTC induction is environmentally regulated. In this model, (1) the partial retention of scnRNA-associated small IESs may reduce the number of productive gene copies that are highly expressed in the parental generation, whereas (2) the more accurate excision of iesRNA-dependent large IESs may increase the copy number of productive genes that are weakly expressed in the parental generation.

\section{Developmentally Regulated Biological Functions in Paramecium}

Next, we asked whether the epigenetically regulated changes in IES recognition/excision efficiency target specific biological functions. We first examined the function of genes that harbor small DCL2/3-sensitive, PTC-inducing, and exon-mapping IESs and are highly expressed at standard conditions (19 genes). 

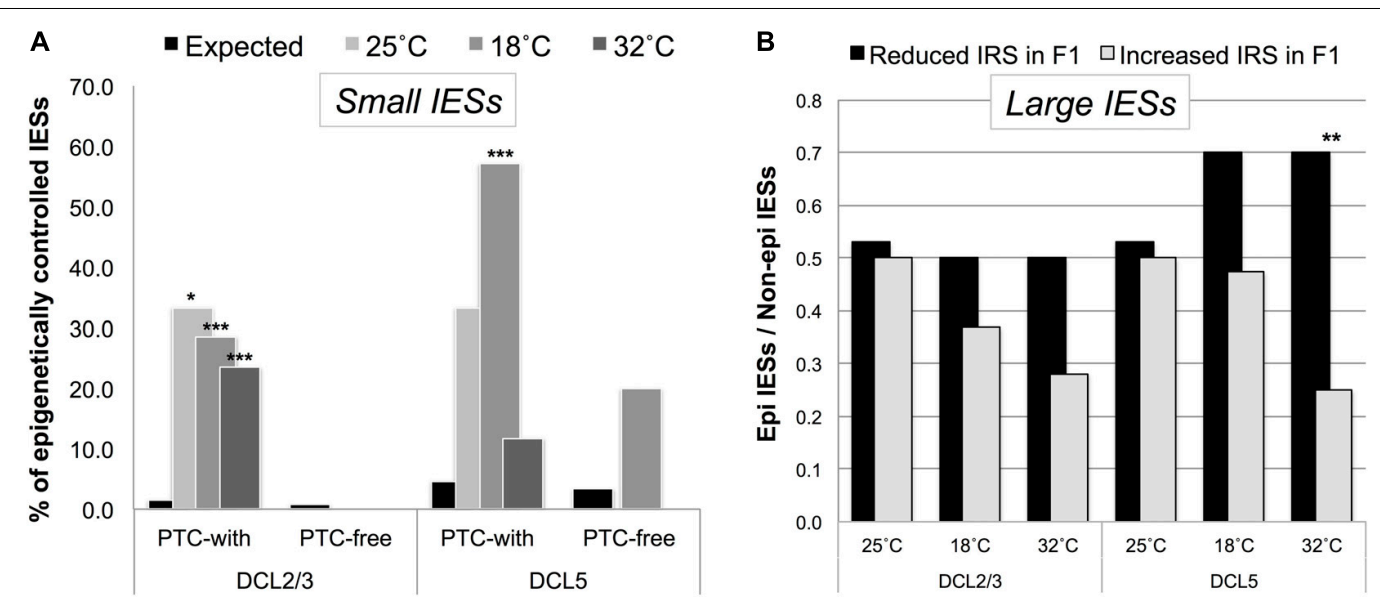

FIGURE 5 | Putative regulated/regulatory IESs fall into a class of IESs that are epigenetically controlled in the P. tetraurelia stock 51 . (A) F1 somatic genomes contain an excess of DCL2/3-sensitive (scnRNA-associated) small exon-mapping and premature-termination codon (PTC)-inducing IESs in genes that are highly expressed in the parental generation. The bars illustrate the fraction of epigenetically regulated IESs in the sets of incompletely excised IESs (IRS $>0.1$ ) detected in F1 cells cultivated at 25,18 , and $32^{\circ} \mathrm{C}$ (Observed), and in the relevant underlying sub-population of IESs with IRS $\geq 0$ (Expected). ${ }^{\star} P<0.05$; ${ }^{\star \star \star} P<0.0001$. (B) An excess of DCL5-sensitive large exon-mapping IESs ( $\left.{ }^{\star \star} P<0.01\right)$ undergo reduced levels of incomplete excision [reduced IES Retention Score (IRS)] in F1 cells cultivated at $32^{\circ} \mathrm{C}$. The parental FO cells are shared across all the sets of $\mathrm{F} 1$ cells and are cultivated at $25^{\circ} \mathrm{C}$.

Despite this gene set's very small size, we detected an overrepresentation of genes involved in aminoacyl-tRNA biosynthesis (Fisher's exact test, $P=0.003$ ). This raises the possibility that PDE may specifically attenuate translation, a classic signature of cellular stress response. We also detected an excess of genes with metalloprotease activity (Fisher's exact test, $P<0.001$ ), which are known to regulate growth factors (Massagué and Pandiella, 1993). If the enhanced retention of small IESs truly reduces the expression of metalloproteases, then one might expect that growth factors' activation (and thus cell growth) is hindered. It is worth noting that, reduced cell growth in Paramecium is coupled with an increased tolerance to stress (Thind et al., 2020).

We then turned to genes that are weakly expressed at standard conditions and that harbor large, DCL5-sensitive, PTC-inducing, and exon-mapping IESs (203 genes). We detected an overrepresentation of genes encoding proteins that are integral components of membrane (Fisher's exact test, $P=5.3 \mathrm{E}-10$ ), involved in import, cyclic nucleotide biosynthetic process, and intracellular signal transduction (Fisher's exact test, $P<0.01$ ), metal ion binding and ion channel activity (Fisher's exact test, $P<0.05)$. Protein domains such as Insulin-like growth factor binding and Epidermal growth factor-like are amongst the most highly over-represented (Fisher's exact test, $P<8.5 \mathrm{E}-12$ ).

Taken together, these enrichment analyses indicate that environmentally induced plasticity in Paramecium may recalibrate stress sensing and responses to environmental conditions (e.g., nutrients).

\section{Parental Experience Contributes to Non-random Developmental Plasticity}

Finally, we considered whether directional changes in PDEmediated somatic variation result from conditions that Paramecium experiences prior to, rather than during, the phase of somatic development. To gain first insights into this question, we examined the somatic genome of F1 Paramecium cells, which were collected in a previous experiment (Vitali et al., 2019) but to date have remained unexplored. Like the F1 lines described above $\left(18^{\circ} \mathrm{C}_{F 1}, 25^{\circ} \mathrm{C}_{F 1}, 32^{\circ} \mathrm{C}_{F 1}\right)$, this additional $\mathrm{F} 1$ line (henceforth referred to as $25^{\circ} \mathrm{C}_{F 1}^{*}$ ) originates from parental, daily re-isolated cells grown at $25^{\circ} \mathrm{C}$. Unlike the other F1 cells, however, the parental line of $25^{\circ} \mathrm{C}^{*} \mathrm{~F} 1$ was additionally subjected to a $40^{\circ} \mathrm{C}$ heat shock for $30 \mathrm{~s}$ daily during vegetative life. Thus, we are in a position to compare the IES retention profile of two sister lines $\left(25^{\circ} \mathrm{C}_{F 1}\right.$ and $\left.25^{\circ} \mathrm{C}_{F 1}^{*}\right)$ that experienced the same temperature during development $\left(25^{\circ} \mathrm{C}\right)$ but originate from isogenic parental cells that experienced different ecological conditions during their vegetative life (constant 25 vs. $25^{\circ} \mathrm{C}$ with daily $40^{\circ} \mathrm{C}$ shock for $30 \mathrm{~s}$ ).

First, we compared the incomplete IES excision profiles of the $25^{\circ} \mathrm{C}_{F 0}, 25^{\circ} \mathrm{C}_{F 1}$, and $25^{\circ} \mathrm{C}^{*}{ }_{F 1}$ mass-cultured cells (Figure 6). Strikingly, this analysis reveals an excess of somatic IESs in the $25^{\circ} \mathrm{C}_{F 1}^{*}$ line similar to that reported for the $18^{\circ} \mathrm{C}_{F 1}$ and $32^{\circ} \mathrm{C}_{F 1}$ lines (Vitali et al., 2019). This observation suggests that the ecological conditions that Paramecium cells experience during their vegetative life can impact the phenotype of their sexual offspring.

Further, the count of incompletely excised small and exonmapping IESs increases for the $25^{\circ} \mathrm{C}_{F 1}^{*}$ line as it does for the $18^{\circ} \mathrm{C}_{F 1}$ and $32^{\circ} \mathrm{C}_{F 1}$ lines, albeit to a lesser extent (Supplementary Figure 1) (F1/F0 ratio: 2.4 and 1.2 for small IESs and large IESs, respectively). $25^{\circ} \mathrm{C}^{*}{ }_{1}$ cells contain also an excess of incompletely excised small IESs (IRS $>0.1$ ) relative to large IESs in genes that are highly expressed at $25^{\circ} \mathrm{C}$ (two-proportion Z-test, $P_{\text {one-tailed }}=0.022$ ), with over $80 \%$ of these IESs disrupting ORF upon retention. Likewise, the magnitude of incompletely excised large and exon-mapping IESs decreases in the $25^{\circ} \mathrm{C}^{*}{ }_{F 1}$ line, as it does for the $18^{\circ} \mathrm{C}_{F 1}$ and $32^{\circ} \mathrm{C}_{F 1}$ lines (Supplementary 


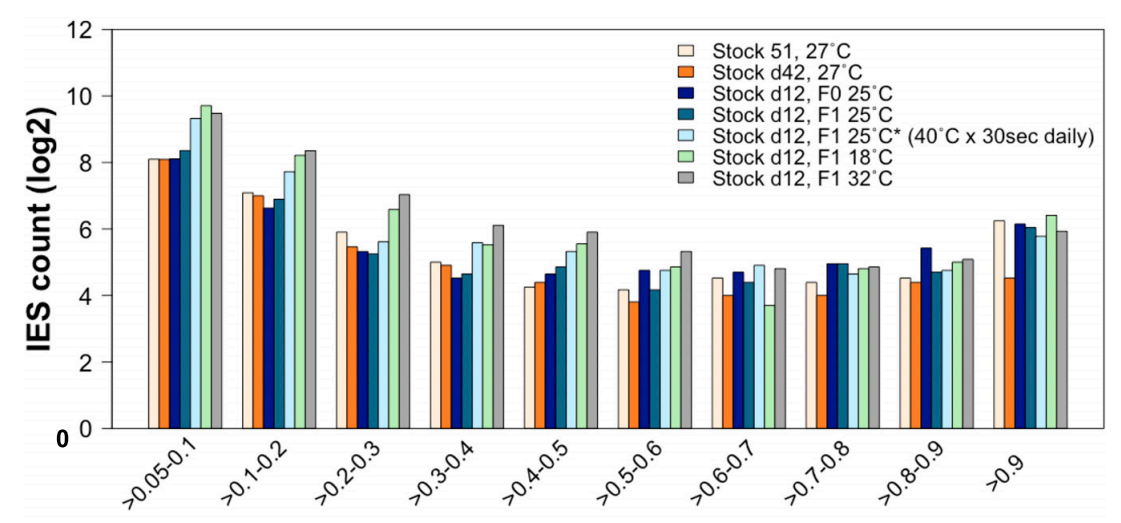

IES Retention Score

FIGURE 6 | The rate of incomplete IES excision is affected by the ecological conditions to which the parental generation is exposed during vegetative life. Compared to lines that have undergone autogamy at $25-27^{\circ} \mathrm{C}$, the count (in log2) of incompletely excised IESs is larger for several IRS classes not only in cells that have undergone autogamy at 18 and $32^{\circ} \mathrm{C}$ but also in cells that have undergone autogamy at $25^{\circ} \mathrm{C}$ but have been grown at $25^{\circ} \mathrm{C}$ and exposed daily to $40^{\circ} \mathrm{C}$ for $30 \mathrm{~s}$.

Figure 2). The magnitude of the IRS reduction is statistically significant compared to the parental cells cultivated at $25^{\circ} \mathrm{C}$ (Wilcoxon rank sum test, $P=0.0009$ ). There is also a tendency of large exon-mapping IESs with significantly reduced IRS in $\mathrm{F} 1$ cells to reside in genes that are weakly expressed in the parental environment $\left(25^{\circ} \mathrm{C}^{*}{ }_{F 1}\right.$ : $6 / 6$ with reduced IRS vs. $1 / 2$ with increased IRS). These findings suggest that the vegetative life experience of the parental cells contributes to the directionality of the next developmental program.

Finally, we inspected how many IESs with IRS $>0.1$ overlap exclusively between the $18^{\circ} \mathrm{C}_{F 1}, 32^{\circ} \mathrm{C}_{F 1}$, and $25^{\circ} \mathrm{C}^{*}{ }_{F 1}$ lines. We detected 11 somatic IESs, of which 10 small and intragenic (a significant excess compared to large IESs; two-proportion Z-test, $P=0.001$ ) (Supplementary Table 1). The $18^{\circ} \mathrm{C}_{F 1}, 32^{\circ} \mathrm{C}_{F 1}$, and $25 C^{*}{ }_{F 1}$ lines share also IESs with significantly reduced IRS (18; 11 intragenic, Supplementary Table 2) and significantly increased IRS (12; 6 intragenic, Supplementary Table 3) relative to the parental line.

These observations suggest that different environmental changes during/prior to somatic development induce nonrandom molecular dynamics in Paramecium. They imply that the newly developing somatic nucleus has access to predictive cues.

\section{CONCLUSION}

Although adaptive plasticity is currently studied only in multicellular species (Gluckman et al., 2009; Beldade et al., 2011; Bateson et al., 2014; Lea et al., 2017; Lu et al., 2019), plasticity can also unfold in unicellular systems like ciliates, which have a stable differentiation between germ line and soma. Previous studies have shown that the ciliate Paramecium experiences plasticity when grown in standard laboratory conditions (Arnaiz et al., 2012; Catania et al., 2013), and more conspicuously upon environmental change (Vitali et al., 2019). Here, we extend our current understanding of the molecular events that unfold during somatic development and the interdependence between these processes and the external environment.

Based on our findings, we propose that alternative DNAlevel splicing in Paramecium may not only reflect errors or snapshots of a mutual process of germ line/somatic DNA sequence conversion (Arnaiz et al., 2012; Catania et al., 2013, 2020), but also regulatory events that are informed by environmental conditions, in line with previous observations (Nowacki et al., 2010; Cervantes et al., 2013; Singh et al., 2014). In this sense, alternative DNA-level splicing in Paramecium is reminiscent of the eukaryotic process of alternative RNA-level splicing (Lewis et al., 2003; Catania and Lynch, 2008; Kelemen et al., 2013; Catania and Schmitz, 2015; Bush et al., 2017; Saudemont et al., 2017).

While waiting for experimental work to determine the hypothesized functional role of IES retention, our observations suggest a cross-generational model for how adaptation to environmental changes may take place in Paramecium. Under this model, the exposure of vegetative cells to new environments mediates a reconfiguration of the epigenetic/transcriptional profile, which may improve the fit between these cells' phenotype and their environment. At the next sexual cycle, this non-genetic reconfiguration recalibrates the trajectory of somatic development, guiding the epigenetic processes that regulate IES excision. This yields a filial somatic macronucleus where non-random developmental plasticity tunes the number of productive somatic gene copies to the new environment, promoting adaptive physiological alterations. Genetic changes that hardwire these epigenetic/physiological changes might follow.

The predictions that this model generates on the relationship between plasticity and evolution can be tested in single-celled ciliates as well as in multicellular systems, where germ line sequences that mobilize in response to stress may be viewed as the equivalent of IES retention/excision in Paramecium. While the hypothesized adaptive developmental program is conceivably an ancestrally selected feature, there is no reason to expect 
that it is hindered in genetically homogeneous populations. Another prediction is that the environmental changes that can induce the adaptive developmental program may not have been experienced in the evolutionary past - we deem it unlikely that Paramecium experienced daily $30 \mathrm{~s}$ shock pulses at $40^{\circ} \mathrm{C}$ during its evolutionary history. We propose that different changes in the environment are capable of inducing similarly, albeit to different degrees, the adaptive developmental program that evolved to help Paramecium cope with changing conditions.

In sum, our findings provide insights into the epigenetic regulation of PDE and plasticity in the single-celled Paramecium. They suggest that conditions experienced by the previous sexual generation(s) may impact the response to current ecological changes, consistent with previous studies and interpretations in long-lived animals, including humans (Kuzawa, 2005; Zipple et al., 2019). These observations call for future studies to explore models where adaptive phenotypes do not solely result from the interplay between genes and current environment, but also account for the epigenetically controlled transfer of ecological information across generations.

\section{DATA AVAILABILITY STATEMENT}

The datasets presented in this study can be found in online repositories. The names of the repository/repositories and accession number(s) can be found below: https://www.ebi.ac.uk/ ena, PRJEB28697, ERR501376, ERR138450, and ERR138952.

\section{REFERENCES}

Arking, R. (2018). Biology of Longevity and Aging: Pathways and Prospects. New York, NY: Oxford University Press.

Arnaiz, O., Mathy, N., Baudry, C., Malinsky, S., Aury, J. M., Denby Wilkes, C., et al. (2012). The Paramecium Germline genome provides a niche for intragenic parasitic DNA: evolutionary dynamics of internal eliminated sequences. PLoS Genet. 8:e1002984. doi: 10.1371/journal.pgen.1002984

Arnaiz, O., and Sperling, L. (2011). ParameciumDB in 2011: new tools and new data for functional and comparative genomics of the model ciliate Paramecium tetraurelia. Nucleic Acids Res. 39, D632-D636. doi: 10.1093/nar/gkq918

Arnaiz, O., Van Dijk, E., Betermier, M., Lhuillier-Akakpo, M., de Vanssay, A., Duharcourt, S., et al. (2017). Improved methods and resources for paramecium genomics: transcription units, gene annotation and gene expression. BMC Genomics 18:483. doi: 10.1186/s12864-017-3887-z

Bateson, P., Gluckman, P., and Hanson, M. (2014). The biology of developmental plasticity and the Predictive Adaptive Response hypothesis. J. Physiol. Lond. 592, 2357-2368. doi: 10.1113/jphysiol.2014.271460

Beldade, P., Mateus, A. R., and Keller, R. A. (2011). Evolution and molecular mechanisms of adaptive developmental plasticity. Mol. Ecol. 20, 1347-1363. doi: 10.1111/j.1365-294X.2011.05016.x

Betermier, M., and Duharcourt, S. (2014). Programmed Rearrangement in Ciliates: Paramecium. Microbiol. Spectr. 2, 1-20. doi: 10.1128/microbiolspec.MDNA30035-2014

Bouhouche, K., Gout, J. F., Kapusta, A., Betermier, M., and Meyer, E. (2011). Functional specialization of Piwi proteins in Paramecium tetraurelia from posttranscriptional gene silencing to genome remodelling. Nucleic Acids Res. 39, 4249-4264. doi: 10.1093/nar/gkq1283

Bush, S. J., Chen, L., Tovar-Corona, J. M., and Urrutia, A. O. (2017). Alternative splicing and the evolution of phenotypic novelty. Philos. Trans. R. Soc. Lond. B Biol. Sci. 372:20150474. doi: 10.1098/rstb.2015.0474

Catania, F., Hagen, R., and Vitali, V. (2020). Prolonged exposure to constant environmental conditions prompts nonrandom genetic variation. bioRxiv [Preprint] doi: 10.1101/2020.04.07.030270

\section{AUTHOR CONTRIBUTIONS}

$\mathrm{RH}$ performed the data acquisition, carried out computational analysis, and wrote the manuscript. VV performed the data acquisition and wrote the manuscript. FC conceived the study, carried out computational analysis, supervised, wrote the manuscript, and secured funding. All authors contributed to the article and approved the submitted version.

\section{FUNDING}

This work was supported by the Deutsche Forschungsgemeinschaft (DFG, German Science Foundation) (281125614/GRK 2220).

\section{ACKNOWLEDGMENTS}

We would like to thank the DFG Research Training Group 2220 "Evolutionary Processes in Adaptation and Disease" at the University of Münster.

\section{SUPPLEMENTARY MATERIAL}

The Supplementary Material for this article can be found online at: https://www.frontiersin.org/articles/10.3389/fcell.2020. 584219/full\#supplementary-material

Catania, F., and Lynch, M. (2008). Where do introns come from? PLoS Biol. 6:e283. doi: 10.1371/journal.pbio.0060283

Catania, F., McGrath, C. L., Doak, T. G., and Lynch, M. (2013). Spliced DNA sequences in the Paramecium germline: their properties and evolutionary potential. Genome Biol. Evol. 5, 1200-1211. doi: 10.1093/gbe/ evt087

Catania, F., and Schmitz, J. (2015). On the path to genetic novelties: insights from programmed DNA elimination and RNA splicing. Wiley Interdiscip. Rev. RNA 6, 547-561. doi: 10.1002/wrna.1293

Cervantes, M. D., Hamilton, E. P., Xiong, J., Lawson, M. J., Yuan, D., Hadjithomas, M., et al. (2013). Selecting one of several mating types through gene segment joining and deletion in Tetrahymena thermophila. PLoS Biol. 11:e1001518. doi: 10.1371/journal.pbio.1001518

Cole, S. W., Conti, G., Arevalo, J. M., Ruggiero, A. M., Heckman, J. J., and Suomi, S. J. (2012). Transcriptional modulation of the developing immune system by early life social adversity. Proc. Natl. Acad. Sci. U.S.A. 109, 20578-20583. doi: $10.1073 /$ pnas.1218253109

Coyne, R. S., Chalker, D. L., and Yao, M. C. (1996). Genome downsizing during ciliate development: nuclear division of labor through chromosome restructuring. Annu. Rev. Genet. 30, 557-578. doi: 10.1146/annurev.genet.30. 1.557

Coyne, R. S., Lhuillier-Akakpo, M., and Duharcourt, S. (2012). RNAguided DNA rearrangements in ciliates: is the best genome defence a good offence? Biol. Cell 104, 309-325. doi: 10.1111/boc.20110 0057

Denby Wilkes, C., Arnaiz, O., and Sperling, L. (2016). ParTIES: a toolbox for Paramecium interspersed DNA elimination studies. Bioinformatics 32, 599-601. doi: 10.1093/bioinformatics/btv691

Duempelmann, L., Skribbe, M., and Bühler, M. (2020). Small RNAs in the transgenerational inheritance of epigenetic information. Trends Genet. 36, 203-214. doi: 10.1016/j.tig.2019.12.001

Duharcourt, S., Butler, A., and Meyer, E. (1995). Epigenetic self-regulation of developmental excision of an internal eliminated sequence on Paramecium tetraurelia. Genes Dev. 9, 2065-2077. doi: 10.1101/gad.9.16.2065 
Duharcourt, S., Keller, A. M., and Meyer, E. (1998). Homology-dependent maternal inhibition of developmental excision of internal eliminated sequences in Paramecium tetraurelia. Mol. Cell. Biol. 18, 7075-7085. doi: 10.1128/mcb.18. 12.7075

Duharcourt, S., Lepere, G., and Meyer, E. (2009). Developmental genome rearrangements in ciliates: a natural genomic subtraction mediated by noncoding transcripts. Trends Genet. 25, 344-350. doi: 10.1016/j.tig.2009.05.007

Duret, L., Cohen, J., Jubin, C., Dessen, P., Gout, J. F., Mousset, S., et al. (2008). Analysis of sequence variability in the macronuclear DNA of Paramecium tetraurelia: a somatic view of the germline. Genome Res. 18, 585-596. doi: 10.1101/gr.074534.107

Ferro, D., Lepennetier, G., and Catania, F. (2015). Cis-acting signals modulate the efficiency of programmed DNA elimination in Paramecium tetraurelia. Nucleic Acids Res. 43, 8157-8168. doi: 10.1093/nar/gkv843

Ghalambor, C. K., Hoke, K. L., Ruell, E. W., Fischer, E. K., Reznick, D. N., and Hughes, K. A. (2015). Non-adaptive plasticity potentiates rapid adaptive evolution of gene expression in nature. Nature 525, 372-375. doi: 10.1038/ nature 15256

Gluckman, P. D., Hanson, M. A., Bateson, P., Beedle, A. S., Law, C. M., Bhutta, Z. A., et al. (2009). Towards a new developmental synthesis: adaptive developmental plasticity and human disease. Lancet 373, 1654-1657. doi: 10. 1016/S0140-6736(09)60234-8

Gout, J. F., Kahn, D., and Duret, L. (2010). The relationship among gene expression, the evolution of gene dosage, and the rate of protein evolution. PLoS Genet. 6:e1000944. doi: 10.1371/journal.pgen.1000944

Huang, D. W., Sherman, B. T., and Lempicki, R. A. (2009a). Bioinformatics enrichment tools: paths toward the comprehensive functional analysis of large gene lists. Nucleic Acids Res. 37, 1-13. doi: 10.1093/nar/gkn923

Huang, D. W., Sherman, B. T., and Lempicki, R. A. (2009b). Systematic and integrative analysis of large gene lists using DAVID bioinformatics resources. Nat. Protoc. 4, 44-57. doi: 10.1038/nprot.2008.211

Jaillon, O., Bouhouche, K., Gout, J. F., Aury, J. M., Noel, B., Saudemont, B., et al. (2008). Translational control of intron splicing in eukaryotes. Nature 451, 359-362. doi: 10.1038/nature06495

Kelemen, O., Convertini, P., Zhang, Z., Wen, Y., Shen, M., Falaleeva, M., et al. (2013). Function of alternative splicing. Gene 514, 1-30. doi: 10.1016/j.gene. 2012.07.083

Kuzawa, C. W. (2005). Fetal origins of developmental plasticity: are fetal cues reliable predictors of future nutritional environments? Am. J. Hum. Biol. 17, 5-21. doi: 10.1002/ajhb.20091

Lea, A. J., Tung, J., Archie, E. A., and Alberts, S. C. (2017). Developmental plasticity: bridging research in evolution and human health. Evol. Med. Public Health 2017, 162-175. doi: 10.1093/emph/eox019

Lepere, G., Nowacki, M., Serrano, V., Gout, J. F., Guglielmi, G., Duharcourt, S., et al. (2009). Silencing-associated and meiosis-specific small RNA pathways in Paramecium tetraurelia. Nucleic Acids Res. 37, 903-915. doi: 10.1093/nar/ gkn 1018

Lewis, B. P., Green, R. E., and Brenner, S. E. (2003). Evidence for the widespread coupling of alternative splicing and nonsense-mediated mRNA decay in humans. Proc. Natl. Acad. Sci. U.S.A. 100, 189-192. doi: 10.1073/pnas. 0136770100

Lhuillier-Akakpo, M., Frapporti, A., Denby Wilkes, C., Matelot, M., Vervoort, M., Sperling, L., et al. (2014). Local Effect of Enhancer of Zeste-Like Reveals Cooperation of Epigenetic and cis-Acting Determinants for Zygotic Genome Rearrangements. PLoS Genet. 10:e1004665. doi: 10.1371/journal.pgen.1004665

Long, H. A., Doak, T. G., and Lynch, M. (2018). Limited mutation-rate variation within the Paramecium aurelia species complex. G3 8, 2523-2526. doi: 10.1534/ g3.118.200420

Lu, A., Petrullo, L., Carrera, S., Feder, J., Schneider-Crease, I., and Snyder-Mackler, N. (2019). Developmental responses to early-life adversity: evolutionary and mechanistic perspectives. Evol. Anthropol. 28, 249-266. doi: 10.1002/evan. 21791

Massagué, J., and Pandiella, A. (1993). Membrane-anchored growth factors. Annu. Rev. Biochem. 62, 515-541. doi: 10.1146/annurev.bi.62.070193.002503

Nettle, D., and Bateson, M. (2015). Adaptive developmental plasticity: what is it, how can we recognize it and when can it evolve? Proc. Biol. Sci. 282:20151005. doi: $10.1098 / \mathrm{rspb} .2015 .1005$
Nowacki, M., Haye, J. E., Fang, W., Vijayan, V., and Landweber, L. F. (2010). RNAmediated epigenetic regulation of DNA copy number. Proc. Natl. Acad. Sci. U.S.A. 107, 22140-22144. doi: 10.1073/pnas.1012236107

R Core Team (2018). R: A Language and Environment for Statistical Computing. Vienna: R Foundation for Statistical Computing.

Sandoval, P. Y., Swart, E. C., Arambasic, M., and Nowacki, M. (2014). Functional diversification of Dicer-like proteins and small RNAs required for genome sculpting. Dev. Cell 28, 174-188. doi: 10.1016/j.devcel.2013.12.010

Saudemont, B., Popa, A., Parmley, J. L., Rocher, V., Blugeon, C., Necsulea, A., et al. (2017). The fitness cost of mis-splicing is the main determinant of alternative splicing patterns. Genome Biol. 18:208. doi: 10.1186/s13059-017-1344-6

Scoville, A. G., and Pfrender, M. E. (2010). Phenotypic plasticity facilitates recurrent rapid adaptation to introduced predators. Proc. Natl. Acad. Sci. U.S.A. 107, 4260-4263. doi: 10.1073/pnas.0912748107

Siegel, R. W. (1961). Nuclear differentiation and transitional cellular phenotypes in the life cycle of Paramecium. Exp. Cell Res. 24, 6-20. doi: 10.1016/00144827(61)90242-7

Singh, D. P., Saudemont, B., Guglielmi, G., Arnaiz, O., Gout, J. F., Prajer, M., et al. (2014). Genome-defence small RNAs exapted for epigenetic mating-type inheritance. Nature 509, 447-452. doi: 10.1038/nature13318

Smith-Sonneborn, J. (1981). Genetics and aging in protozoa. Int. Rev. Cytol. 73, 319-354. doi: 10.1016/s0074-7696(08)61291-7

Sommer, R. J. (2020). Phenotypic plasticity: from theory and genetics to current and future challenges. Genetics 215, 1-13. doi: 10.1534/genetics.120.303163

Sung, W., Tucker, A. E., Doak, T. G., Choi, E., Thomas, W. K., and Lynch, M. (2012). Extraordinary genome stability in the ciliate Paramecium tetraurelia. Proc. Natl. Acad. Sci. U.S.A. 109, 19339-19344. doi: 10.1073/pnas.1210663109

Swart, E. C., Denby Wilkes, C., Sandoval, P. Y., Hoehener, C., Singh, A., Furrer, D. I., et al. (2017). Identification and analysis of functional associations among natural eukaryotic genome editing components [version 1; referees: 1 approved, 1 approved with reservations]. F1000Research 6:1374. doi: 10.12688/ f1000research.12121.1

Takagi, Y., Suzuki, T., and Shimada, C. (1987). Isolation of a Paramecium tetraurelia mutant with short clonal life-span and with novel life-cycle features. Zool. Sci. 4, 73-80.

Thind, A. S., Vitali, V., Guarracino, M. R., and Catania, F. (2020). What's genetic variation got to do with it? Starvation-induced self-fertilization enhances survival in Paramecium. Genome Biol. Evol. 12, 626-638. doi: 10.1093/gbe/ evaa052

Tung, J., Zhou, X., Alberts, S. C., Stephens, M., and Gilad, Y. (2015). The genetic architecture of gene expression levels in wild baboons. eLife 4:04729. doi: 10 . 7554/eLife.04729

Vitali, V., Hagen, R., and Catania, F. (2019). Environmentally induced plasticity of programmed DNA elimination boosts somatic variability in Paramecium tetraurelia. Genome Res. 29, 1693-1704. doi: 10.1101/gr.2453 32.118

Watve, M. (2017). Developmental plasticity: need to go beyond naive thinking. Evol. Med. Public Health 2017, 178-180. doi: 10.1093/emph/eox020

Wells, J. C. (2012). A critical appraisal of the predictive adaptive response hypothesis. Int. J. Epidemiol. 41, 229-235. doi: 10.1093/ije/dyr239

Wells, J. C. K. (2017). Understanding developmental plasticity as adaptation requires an inter-generational perspective. Evol. Med. Public Health 2017, 185-187. doi: 10.1093/emph/eox023

Zipple, M. N., Archie, E. A., Tung, J., Altmann, J., and Alberts, S. C. (2019). Intergenerational effects of early adversity on survival in wild baboons. eLife 8:e47433. doi: 10.7554/eLife.47433

Conflict of Interest: The authors declare that the research was conducted in the absence of any commercial or financial relationships that could be construed as a potential conflict of interest.

Copyright (C) 2020 Hagen, Vitali and Catania. This is an open-access article distributed under the terms of the Creative Commons Attribution License (CC BY). The use, distribution or reproduction in other forums is permitted, provided the original author(s) and the copyright owner(s) are credited and that the original publication in this journal is cited, in accordance with accepted academic practice. No use, distribution or reproduction is permitted which does not comply with these terms. 